\title{
Acceleration of emergence of E. coli antibiotic resistance in a simulated sublethal concentration of copper and tetracycline co-contaminated environment
}

\author{
Jinmei Li ${ }^{1}$, Irfan Ali Phulpoto ${ }^{1}$, Guilong Zhang ${ }^{2}$ and Zhisheng Yu ${ }^{1 *}$
}

\begin{abstract}
An environment co-contaminated with metals and antibiotics ultimately exposes bacteria to these metals and antibiotics simultaneously. This study aims to explore the efficacy of sublethal concentrations of copper ions contaminated with tetracycline regarding antibiotic resistance in a sensitive strain of $E$. coli $\mathrm{K} 12$. The study proved that a copper ions and tetracycline co-contaminated environment could considerably enhance the mutation frequencies of chloramphenicol and polymyxin B resistance in antibiotic susceptible E. coli; however, the equivalent copper ions and tetracycline alone showed weaker effects. Results also demonstrated that an environment co-contaminated with relatively high sublethal concentrations of copper ion and tetracycline co-contaminated environment could induce much higher antibiotic resistance than the low sublethal and control groups. Whole-genome characterization results indicated that variability existed within the genotype and phenotype involved in antibiotic resistance. Additionally, the evolved resistant strains displayed hereditary resistance after 5 round culture cycles in LB broth over 5 days. Results implied that co-contamination with metals and antibiotics environment could strengthen resistance and contribute to the induction and dissemination of antibiotic resistance in metal and antibiotic co-contaminated environment.
\end{abstract}

Keywords: ARG, Heavy metals, Co-contamination, Sub-MIC

\section{Key points}

- Mutation frequencies increased with cross contamination.

- Antibiotic resistance induction was dose dependent.

- Genotype and phenotype could diverge under the same stress condition.

- The resistant mutants displayed hereditary resistance.

\footnotetext{
*Correspondence: yuzs@ucas.ac.cn

${ }^{1}$ College of Resources and Environment, University of Chinese Academy of Sciences, 19 A Yuquan Road, Shijingshan District, Beijing 100049, China Full list of author information is available at the end of the article
}

\section{Introduction}

Antibiotics are the essential double-edged sword: one hand, antibiotics are a powerful weapon to life-saving, on the other hand, misuse and overuse urge bacteria development and dissemination of resistance (Zhu et al. 2013; Lv et al. 2014; Vikesland et al. 2017). Antibiotic resistance forms an increasing risk to our body health, since this is relevant to the therapeutic effects of antibiotics (Vikesland et al. 2017). As the result of over-use of antibiotics induced the selective stress in our environment, more and more antibiotic resistant bacteria (ARB) and antibiotic resistance genes (ARGs) were determined in clinical as well as nature (Alonso et al. 2001). Residual antibiotics from human and animal feces/wastes, hospital waste and pharmaceutical industries eventually contaminate the soil and water environments (Pruden et al. 2006; Nolivos 
et al. 2019; Povolo and Ackermann 2019). Soil fertilizer with animal waste and sewage sludge are causing antibiotic accumulation (Zhu et al. 2013). Residual antibiotics could run away from the soil environment, then get to the water ecosystem (Martinez 2008). Additionally, antibiotics are regarded as "pseudo-persistent", although most antibiotics have relatively short half-lives, their residues persist in the environment (Pan et al. 2011). Even at several 100-fold below the minimal inhibitory concentration of antibiotics could enrich antibiotic resistant bacteria (Gullberg et al. 2011).

However, heavy metals are persistent in nature, gathering in a diverse constituent of the ecosystems ( $\mathrm{Lu}$ et al. 2014). Copper and zinc are generally employed as food additive in animal feed diets through the supplement of the ingredients in their compound feed, exceeding the needs for normal growth of the animals, and for precaution of animal disease, as well as for the growth promotion and medical remedies (Baker-Austin et al. 2006; Hau et al. 2017). Reports claimed that copper in the soil, not only selects for copper resistance but also co-selects for resistance to antibiotics, for example, chloramphenicol, tetracycline and ampicillin (Berg et al. 2005). Most of researches related to antibiotic resistance were focused on high concentrations, such as, more than the minimal inhibitory concentration (MIC)) (Seiler and Berendonk 2012), while the effect of relatively low concentrations, that is sublethal $(<$ MIC) still mainly unclear.

Additionally, sub-inhibitory concentration of metals could endow bacteria antibiotic resistance through co-selection, as well as heavy metal resistance (Gullberg et al. 2014; Chen et al. 2015). Sublethal concentrations of antibiotics induce mutagenesis by stimulating the production of ROS, these effects can result in mutant bacterial that are sensitive to the applied antibiotic but resistant to other antibiotics (Kohanski et al. 2010). As we know, heavy metal pollution in environments could contribute to the maintenance and dissemination of antibiotic resistance (Stepanauskas et al. 2005; Henriques et al. 2016; Zhang et al. 2018a; Imran et al. 2019). Such as, Zhu et al. (2013) reported a significant correlation between copper concentration and the incidence of ARGs (Zhu et al. 2013). In some natural ecosystems, heavy metal and antibiotic co-contaminated together could drive the dissemination of antibiotic resistance in bacteria (BakerAustin et al. 2006; Wang et al. 2014), and co-exposure to zinc and antibiotic co-contaminated environments such as oxytetracycline in activated sludge bioreactors could enhance the resistance of the microbial community towards antibiotics (Peltier et al. 2010). Findings suggest that the low levels of antibiotics and heavy metals present in polluted external environments could allow for selection and enrichment of bacteria with multi-resistance plasmids (Gullberg et al. 2014). But how sub-inhibitory concentration of tetracycline and copper ion contribute to the emergence and maintenance of clinically significant $E$. coli is largely unknow.

Evolutionary experiments aim to determine whether sublethal concentrations of copper ions contaminated with tetracycline could increase the bacterial antibiotic resistance through stimulation in a co-contaminated environment. Whole-genome sequencing analysis will be utilized to gain insight into the potential mechanisms of resistance. Comparative molecular analysis of resistance determinants, coupled with phenotypic analysis, may contribute to further understanding of antibiotic resistance sources. This study's findings will clarify the complicated relationship between heavy metal and antibiotic co-contamination with antibiotic resistance. A summary of the changes in resistance with generations on the evolution cycle in $E$. coli will be examined in a further study.

\section{Materials and methods}

\section{Strains, antibiotics and selection condition}

First, E. coli K12 (MG1655) was been sequenced (Shanghai Majorbio Bio-pharm Technology Co., Ltd), which was designated as the original wild type strain (Table 1). Activating $E$. coli from storage tube with glycerol stock which stored in $-80{ }^{\circ} \mathrm{C}$, expanding propagating on a Luria Bertani (LB) agar plates, cultured at $37^{\circ} \mathrm{C}$ for $16 \mathrm{~h}$. The selected seed strain was cultured in LB broth at $37{ }^{\circ} \mathrm{C}$ for $12 \mathrm{~h}$ for following selected experiments ( $\mathrm{Li}$ et al. 2016).

Table 1 The MIC of each antibiotic to wild-type E. coli K12

\begin{tabular}{|c|c|c|c|c|c|}
\hline No & Antibiotics & Abbreviation & Classification & Stock solution (mg/L) & $\mathrm{MIC}(\mathrm{mg} / \mathrm{L})$ \\
\hline 1 & Ciprofloxacin & Cip & Quinolones & 200 & 0.2 \\
\hline 2 & Tetracycline & Tet & Tetracyclines & 10 & 2.34 \\
\hline 3 & Gentamicin & Gen & Aminoglycosides & 10 & 8.75 \\
\hline 4 & Polymyxin B & Pol & Polypeptides & 10 & 0.94 \\
\hline 5 & Erythromycin & Ery & Macrolides & 6.4 & 15 \\
\hline 6 & Chloramphenicol & $\mathrm{Chl}$ & Chloramphenicols & 30 & 4.69 \\
\hline
\end{tabular}


The involved antibiotics: chloramphenicol (Chl), ciprofloxacin (Cip), erythromycin (Ery), gentamycin (Gen), tetracycline (Tet), and polymyxin $\mathrm{B}$ (Pol) and cupric (CuSO4.5H2O) were get from Solarbio, Inc. (Shanghai, China). 90\% inhibition of growth was regarded as the MIC of each antibiotic, which was determined as Additional file 1: Test S1 described, and the MIC of copper ions was also investigated with the same method. The detailed accounts are displayed in Additional file 1: Text S1. The tetracycline resistant cultures were kept from light so as not to degrade the antibiotic.

\section{Determination of minimum inhibitory concentrations}

The MICs were investigated after 40 sub-culture cycles, using previously described methods (Lv et al. 2014). The serially diluted strain cultures were grown on LB agar plates for $16 \mathrm{~h}$ at $37^{\circ} \mathrm{C}$. Then 10 colonies from each parallel agar plate were unintentionally selected from each sub-culture. These randomly picked strains were cultured in $3 \mathrm{~mL}$ of LB broth for $5-6 \mathrm{~h}$ at $37^{\circ} \mathrm{C}$ to investigate the MICs (Additional file 1: Text S1) (Li et al. 2016). And, the MICs of the original E. coli K12 strain was also determined (Table 1).

\section{Exposure to copper ions and tetracycline environment}

Detailed procedures of the exposure experiments are shown in Fig. 1. A sample, totaling $5 \mathrm{~mL}$, including
$0.5 \mathrm{~mL}$ of original E. coli cultures $\left(10^{6} \mathrm{CFU}\right)$ and $4.5 \mathrm{~mL}$ of fresh LB broth, containing corresponding concentrations of copper ions and tetracycline, was cultured in a $15 \mathrm{~mL}$ sterilized tube at $37^{\circ} \mathrm{C}$ for $24 \mathrm{~h}$. Subsequently, $0.5 \mathrm{~mL}$ of the cultures was transferred into a new $15 \mathrm{~mL}$ sterilized tube with $4.5 \mathrm{~mL}$ fresh LB broth to sub-culture with corresponding copper ions and tetracycline. The original isogenic $\mathrm{K} 12$ was cultured in LB broth; this lacked any copper ions or tetracycline and was used as the control. All experiments were conducted in triplicate.

The exposure dosages of copper ions and tetracycline applied in this research were calculated according to the MICs as well as the concentration of environments (Additional file 1: Fig. S1, Table 1); taking into account the tested and evaluated environmental concentrations (Zhu et al. 2013), examples of which include the cupric concentration in Dawu River, which ranged from 12 to $30 \mathrm{mg} / \mathrm{L}$ (Huang Changgan et al. 2004), also the cupric concentration in previous studies which ranged from 8 to $500 \mathrm{mg} / \mathrm{L}$ (Chen et al. 2015; Poole 2017). Resistant bacteria can be selected at the high concentrations of tetracyclines used therapeutically, but what role the extremely low tetracycline concentration present plays in selection still largely unclear (Gullberg et al. 2011). Thus, the concentration of tetracycline during the experiments was set as $0.0234 \mathrm{mg} / \mathrm{L}$. The resistance evolution experiments were observed in 5 conditions (Fig. 1b), gradually
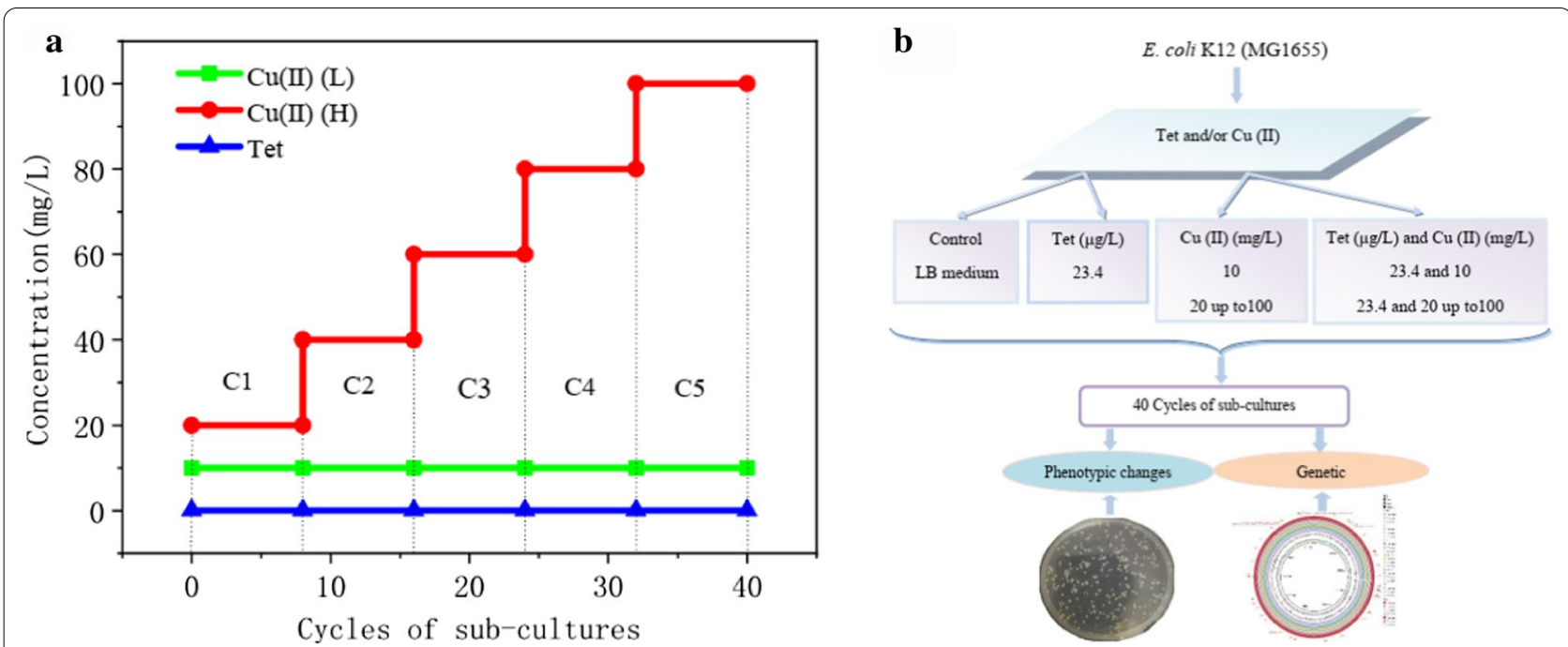

Fig. 1 Diagram exposure sub-culture and schematic experimental design. Antibiotic resistance selection by both copper ion (Cu (II)) and tetracycline (Tet). The initial bacterial culture with copper ions and tetracycline treatments or without any copper ions or tetracycline treatment (control groups) as cycle 0 (recorded as C0), and subsequent were recorded as C1, C2, etc. Each cycle was 8 days, a total of 5 cycles. a Cycles of the exposure sub-culture; $\mathbf{b}$ The concentrations of $\mathrm{Cu}$ (II) and Tet, during 5 cycles of sub-cultures for antibiotic resistance selection with mental ions of $\mathrm{Cu}(\mathrm{II})$ and Tet. The concentration of tetracycline during the experiments was set as $0.0234 \mathrm{mg} / \mathrm{L}$, gradually increasing the copper ion dose concentrations from 1/100 $\times$ MIC $(20 \mathrm{mg} / \mathrm{L})$ up to $1 / 10 \times \mathrm{MIC}(100 \mathrm{mg} / \mathrm{L})$, as the relatively high concentration evolution condition; and relatively lower concentrations of copper ion at about $10 \mathrm{mg} / \mathrm{L}$. H: Strains exposure at high copper ion concentrations; L: Strains exposure at low copper ion concentrations. All experiments were performed in triplicate 
increasing the dose concentrations from $1 / 100 \times$ MIC $(20 \mathrm{mg} / \mathrm{L})$ up to $1 / 10 \times \mathrm{MIC}(100 \mathrm{mg} / \mathrm{L})$, as the relatively high concentration evolution condition; and relatively lower concentrations of copper ions at about $10 \mathrm{mg} / \mathrm{L}$. In the relatively high concentration evolution selection culture, the copper ion concentrations gradually increased from a starting point of $20 \mathrm{mg} / \mathrm{L}$ to approximately $100 \mathrm{mg} / \mathrm{L}$ (recorded as H) (Fig. 1a). In the relatively low concentration evolution cultures, the concentrations of copper ions and tetracycline were kept consistent at $10 \mathrm{mg} / \mathrm{L}$ and $0.0234 \mathrm{mg} / \mathrm{L}$, respectively (recorded as L). All experiments were repeated in triplicate.

The initial bacterial culture with copper ions and tetracycline treatments or without any copper ions and tetracycline (control groups) were recorded as $\mathrm{C} 0$. The following sub-cultures were devised as cycle 1 , cycle 2 , etc., recorded as C1, C2, etc., respectively (Fig. 1a). Four milliliter of every experimental cycle sub-culture was mixed with glycerol solution in a $15 \%(\mathrm{v} / \mathrm{v})$, then stored at $-80{ }^{\circ} \mathrm{C}$ for the subsequent step (Li et al. 2019). All culture steps were incubated with $200 \mathrm{rpm}$ shaking in aerated incubators; each cycle was $24 \mathrm{~h}$, a total of 40 subculture cycles were carried out (Fig. 1a).

\section{Subsequent antibiotic resistance determination}

After 40 cycles of sub-culture, the mutation rates were investigated (Kohanski et al. 2010). To determine the antibiotic resistant mutation rates, sub-cultured strains were streaked on LB agar plates containing corresponding antibiotics (Table 1), following cultured for $48 \mathrm{~h}$ at $37^{\circ} \mathrm{C}$ (Li et al. 2016), and the colonies were counted. The colonies grew on LB agar without any antibiotics were regarded as the total bacterial concentrations. The colonies grew on LB agar plates containing antibiotic were also determined as resistance to the corresponding antibiotics (Lv et al. 2014). The maximum-likelihood method was used to investigate the mutation frequency as previous described (Lv et al. 2014), as the follow formula (1).

$$
\text { Mutation rates }=\frac{\text { Resistant clones }}{\text { Total number of clones }}
$$

Fold changes of mutation rate were measured through each exposure treatment relative to an untreated control group.

\section{Hereditary stability determination}

Hereditary stability of the randomly selected clones after 40 cycles was tested for 5 days of sub-culture cycles (Lv et al. 2014; Zhang et al. 2018b). That is, the selected strains were diluted 1:100 in $5 \mathrm{~mL}$ of fresh LB media without any treatment, then regrowth for $24 \mathrm{~h}$ at $37^{\circ} \mathrm{C}$, $180 \mathrm{rpm}$. Each selected strain was exposed to 5 such cycles of growth (Zhang et al. 2018b). The MIC values were measured after 5 days of sub-culture, then matched the MICs of the initial strains to determine the hereditary stability. The MIC determination method was used as former research described ( $\mathrm{Li}$ et al. 2016; Khan et al. 2017) (Additional file 1: Text S1).

\section{DNA extraction and whole-genome sequencing}

The strains from each treated group and the control group cultured 2 to 3 times on LB agar plates without any antibiotics for $16 \mathrm{~h}$ at $37{ }^{\circ} \mathrm{C}$. The Universal Genomic DNA Extraction Kit (Takara, Beijing, China) was used to extract the total DNA, the manipulation process was depending on the manufacturer's instructions. The concentration and purity were investigated by Nanodrop 2000 (Thermo Fisher Scientific, Wilmington, DE).

The NEXTflex ${ }^{\mathrm{TM}}$ Rapid DNA-Seq Kit was applied to set up Illumina sequencing libraries. Briefly speaking, $5^{\prime}$ prime ends were first end-repaired and phosphorylated. Next, the $3^{\prime}$ ends were A-tailed and ligated to sequencing adapters. The third step was to enrich the adapters-ligated products using PCR (Shanghai Majorbio Bio-pharm Technology Co., Ltd).

\section{Data analysis}

Microsoft Excel 2016 (Microsoft Inc., USA) was used to manipulate the mean, standard deviation and fold change of data. Independent sample t-test was used to analysis significant differences (SPSS 18.0, USA).

\section{Results \\ Copper ion and tetracycline co-contaminations enhance the mutation frequency of polymyxin $B$ and chloramphenicol}

Figure 2 indicates the effects on bacterial antibiotic resistance after exposing the E. coli strains to the sublethal concentrations of copper ion and tetracycline. The mutation rate of the control, which was not treated by sublethal concentrations of copper ion and tetracycline, was regarded as the spontaneous mutation rate, which ranged from $10^{-8}$ to $10^{-6}$ for different antibiotics.

Sublethal concentrations of copper ions and tetracycline co-contaminated treatments could critically enhance the resistant mutation rates of polymyxin $B$ and chloramphenicol $(\mathrm{P}<0.05)$ (Fig. 2a, b), compared with the control groups. The increased mutation rate of resistance to ciprofloxacin and gentamicin was $<$ twofold compared to the control groups (data is not displayed). Sublethal concentrations of copper ions and tetracycline co-contamination resulted in copper dose-dependent alteration in resistance mutation rates for polymyxin B (Fig. 2a) and chloramphenicol (Fig. 2b). The higher the level of copper 

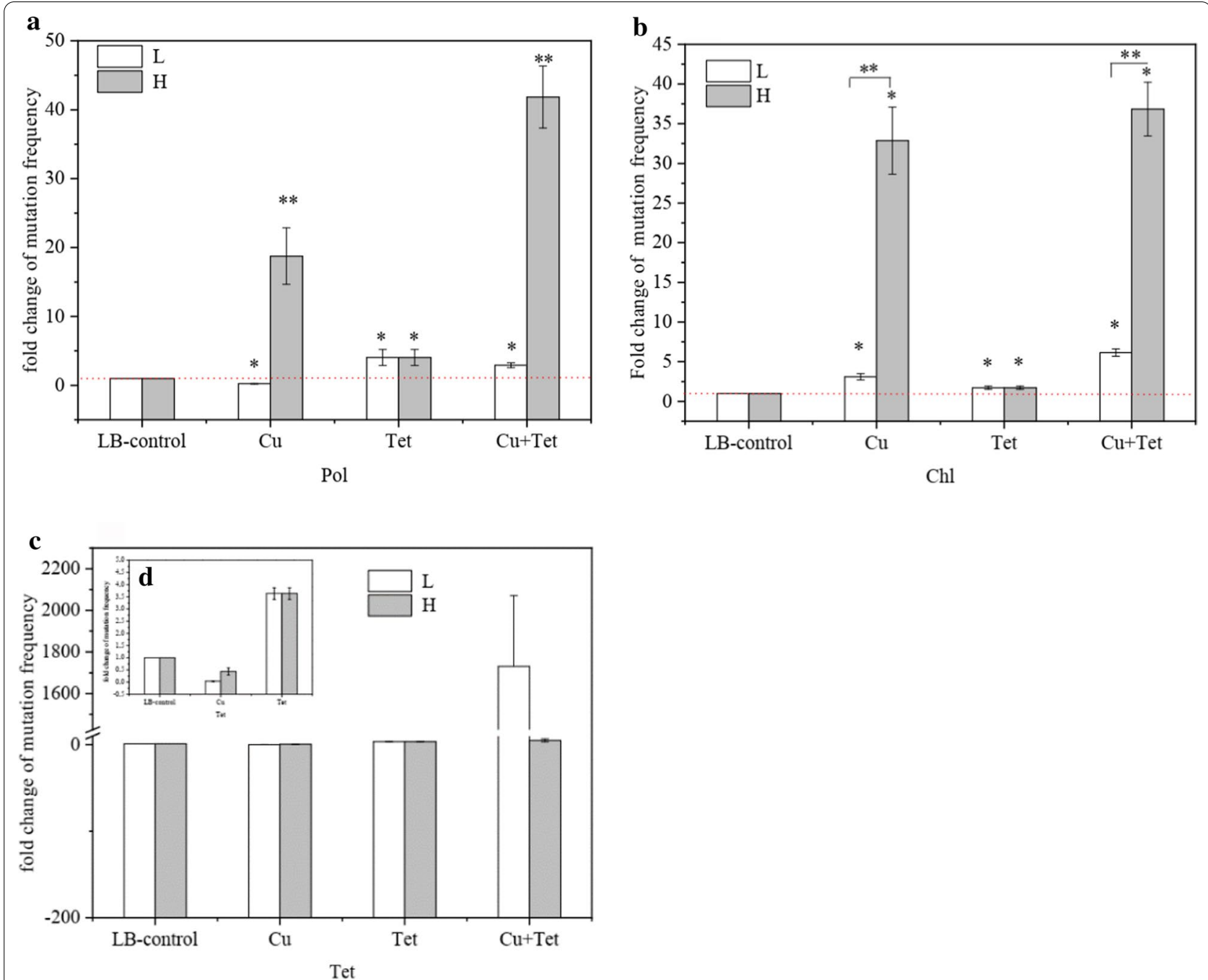

Fig. 2 Mutation frequency changes for a polymyxin B (Pol), b chloramphenicol (Chl) and $\mathbf{c}$, $\mathbf{d}$ tetracycline (Tet) respectively induced by copper ions (Cu) or tetracycline (Tet) and their co-contaminations. LB-control was without any treatment. H: Strains exposure at high copper ion concentrations; $\mathrm{L}$ : Strains exposure at low copper ion concentrations. All experiments were performed in triplicate. All experiments were manipulated in triplicate. Significant differences were tested: ${ }^{*}(P<0.05),{ }^{* *}(P<0.01)$ and ${ }^{* * *}(P<0.001)$

concentration in the compounds, the higher chance of polymyxin B or chloramphenicol resistance; copper ion treatment alone was similar.

The resistance of E. coli $\mathrm{K} 12$ to polymyxin B or chloramphenicol showed that relatively high sublethal concentrations of copper ion, acting alone or in combination with antibiotics, can increase the frequency of mutations by $0.2-2.9,18.8-41.9,3.1-6.1$, and $32.9-36.8$ folds respectively, compared with the controls. However, only treatment with a relatively low sublethal concentration of copper ion and tetracycline co-contamination caused a significant increase in the mutation rates for tetracycline resistance. The increase was 1730 -8-fold; however, there was only a fivefold increase with a relatively high sublethal concentration of copper ion and tetracycline co-contamination (Fig. 2c). Additionally, both low and high sublethal copper ion treatment induced a mutation resistance to tetracycline with a 0.04- and 0.44-fold increase, respectively (Fig. 2c). Resistance to tetracycline increased 3.64-fold with tetracycline treatment (Fig. 2d).

\section{Effects of sublethal concentration of copper ion and tetracycline co-contaminations on antibiotic resistance}

With a sublethal concentration of copper ions and tetracycline exposure, selected strains that demonstrated clinically relevant resistance to ciprofloxacin, erythromycin, polymyxin $\mathrm{B}$, tetracycline, and chloramphenicol 

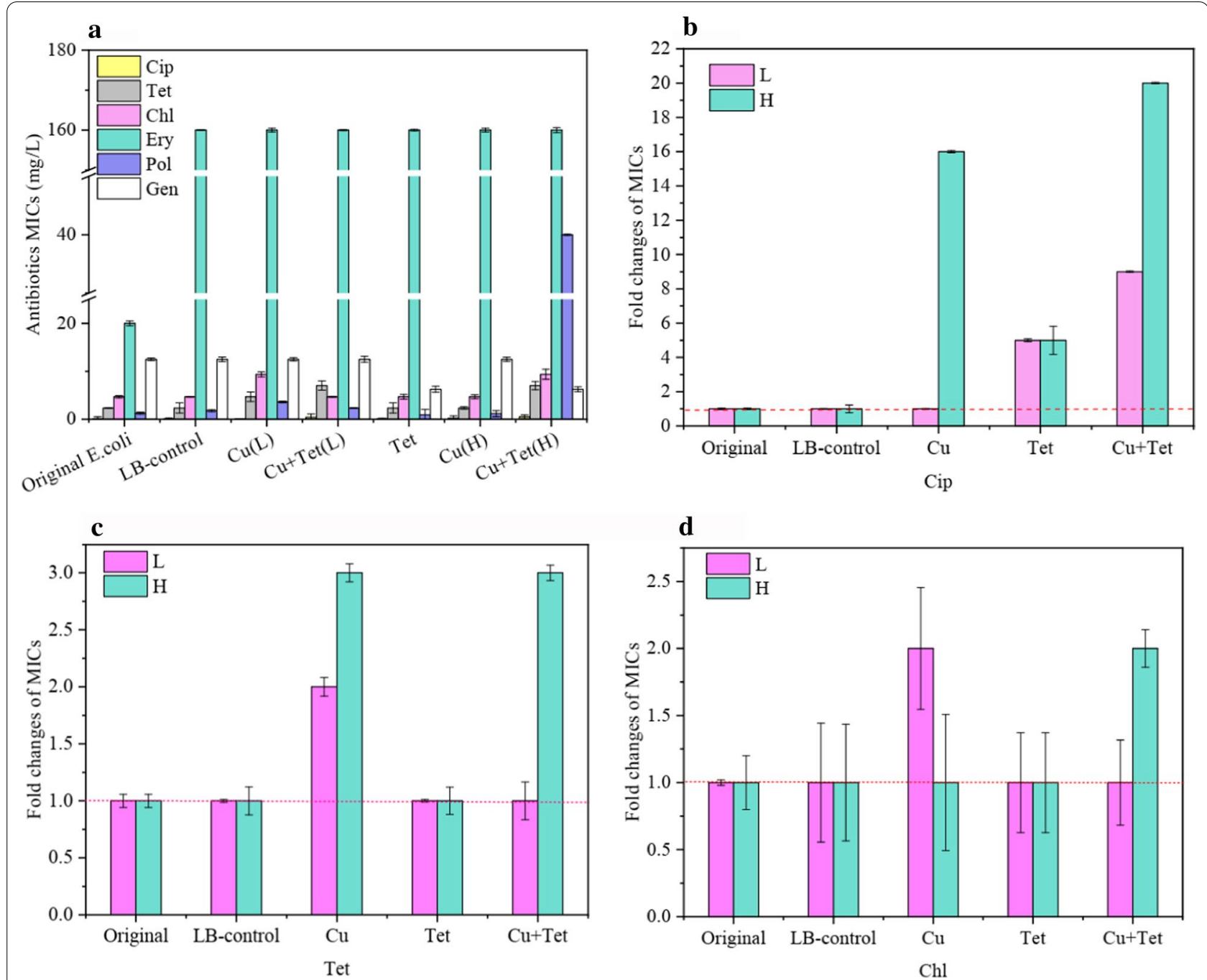

Fig. 3 Change of the minimum inhibition concentrations (MICs). a Ciprofloxacin (Cip), tetracycline (Tet), chloramphenicol (Chl), gentamicin (Gen), polymyxin B (Pol) and erythromycin (Ery)) among the original E. coli K12 in LB medium (LB-control), copper ions and tetracycline alone act or contaminated treatment; fold changes of MICs of ciprofloxacin- (b), tetracycline- (c), and chloramphenicol-resistant (d) induced by copper ion (Cu) and/or tetracycline (Tet) treatment. H: Strains exposure at high copper ion concentrations; L: Strains exposure at low copper ion concentrations

with an increase in MICs by 1 to 32-fold (Fig. 3a). The resistance to gentamycin was no more than a onefold increase in MICs (Fig. 3a). The average MICs for environments co-contaminated with sublethal copper ions and tetracycline-selected ciprofloxacin-resistant strains were more than 11-16 and 16-20 folds higher than that of the treatment with copper ion alone or the original or LB-control E. coli (Fig. 3b). The relatively high sublethal concentration of copper ion contaminated with tetracycline antibiotic-selected resistant strains displayed over fourfold higher MICs than the relatively low sublethal concentration for ciprofloxacin. Tetracycline-selected ciprofloxacin-resistant strains exhibited fivefold higher MICs than the original or LB-control E. coli (Fig. 3b).
The tetracycline and chloramphenicol-resistant strains selected with relatively low sublethal copper ion treatment showed higher resistance than the copper ion and tetracycline co-contaminated environment (Fig. 3c, d).

\section{Whole-genome sequence analysis of the evolution strains}

Whole-genome sequencing analysis of the selected mutants was undertaken to determine the underlying genetic mechanisms involved in antibiotic resistance caused by sublethal of copper ions and tetracycline environments. Two or three isogenic clones were selected from the strains tackled by relatively low or high sublethal concentrations of copper ions and tetracycline for 
whole-genome sequencing analysis (Brockhurst et al. 2011).

Two genetic changes involved in ARG were determined, that is $m d t F$ and vanRI, including a substitution mutation and insertion genetic changes induced by copper ion or tetracycline exposure (Fig. 4 and Table 2). Two different changes were also identified on $m d t F$ and $v a n R I$ genes in the LB-control strain, caused by spontaneous mutations throughout the sub-culture processes, such as growing situation and stress (Table 2). One genetic mutation in 1 gene was detected in $E$. coli strains induced by a relatively high sublethal copper ion environment (Fig. 4 and Table 2). The genetic insertion was associated with the membrane transporter gene $(m d t F)$ for the mdtEF-TolC efflux complex. Similarly, 1 genetic mutation in 1 gene was detected in E. coli strains caused by relatively low sublethal copper ions or tetracycline treatment and relatively low sublethal concentration in their co-contaminated environments; which was associated with a transcriptional activator in the vanSR regulator within the vanI glycopeptide resistance gene cluster (vanRI) (Fig. 4 and Table 2). The number and evolution of clinically relevant antibiotic resistance genes could be induced by contaminations; other genes with main functions, but with a resistance phenotype, are present in the environmental resistome.

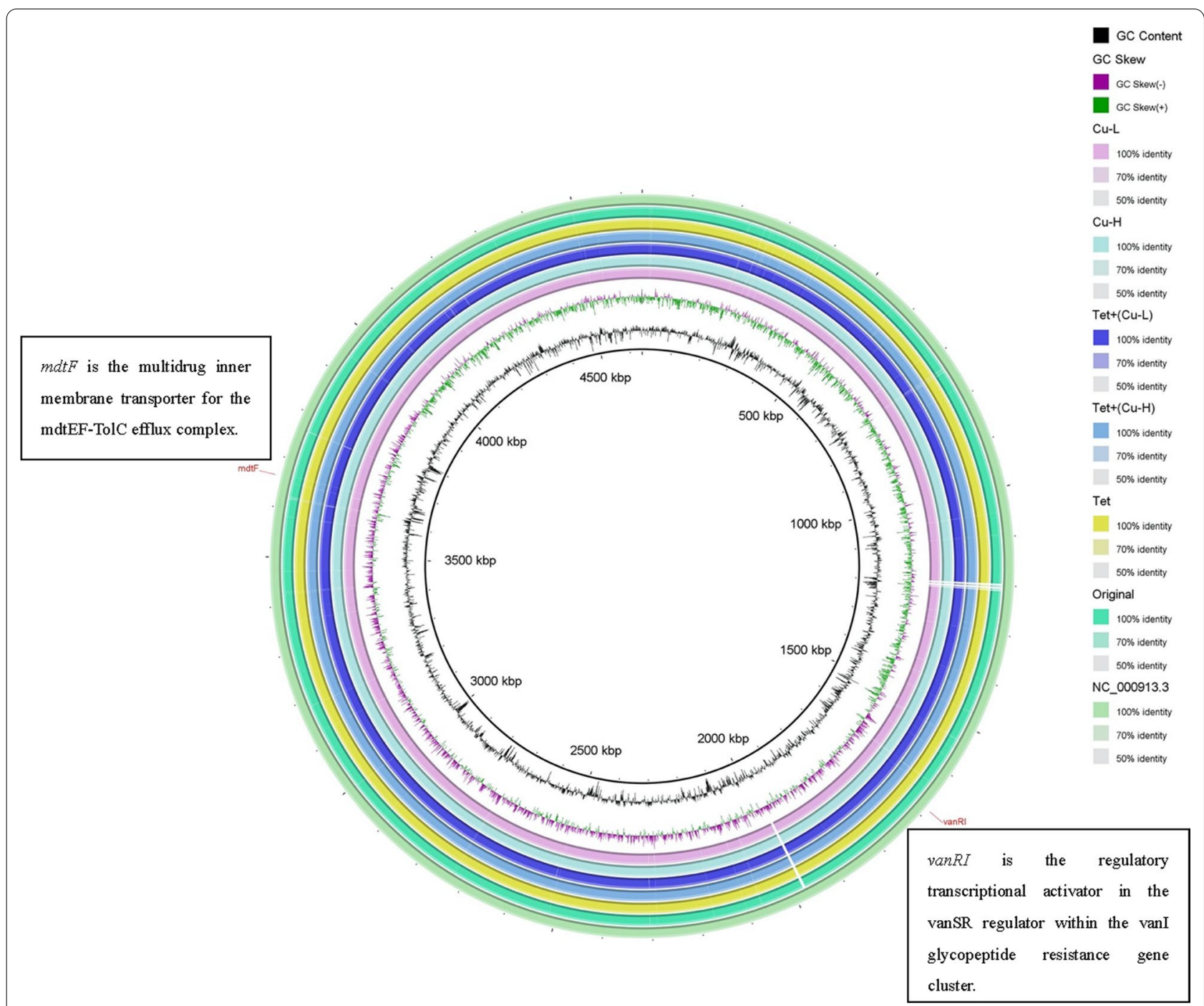

Fig. 4 Comparison of genetic changes of genome coverage and mutations identified in strains selected under exposure to copper ion (Cu) and tetracycline (Tet) concentrations, initial E. coli K12 and control. Two genetic changes were determined, that is $\mathrm{mdtF}$ and vanRI. The detailed information of mutations on these genetic changes was shown in Table 2 
Table 2 Summary of identified genetic changes in untreated control clones selected in LB medium and in strains selected in exposure environments

\begin{tabular}{llll}
\hline Strains names & Site & Types & Gene \\
\hline Original E. coli & No genetic change & & \\
LB-control & $1210-1215$ & TTANGG & mdtF \\
LB-control & 572 & G $\rightarrow$ A & vanRl \\
Cu-H & $1203-1217$ & GATAGGGTTANTGGC & mdtF \\
Cu-L & 572 & G $\rightarrow$ A & vanRl \\
Tet & 572 & $G \rightarrow A$ & vanRl \\
Tet $+(\mathrm{Cu}-\mathrm{L})$ & 572 & $\mathrm{G} \rightarrow \mathrm{A}$ & vanRl \\
Tet $+(\mathrm{Cu}-\mathrm{H})$ & 572 & $\mathrm{G} \rightarrow \mathrm{A}$ & $\operatorname{vanRl}$ \\
\hline
\end{tabular}

The $m d t F$ is the multidrug inner membrane transporter for the mdtEF-TolC efflux complex; van $R I$ is the regulatory transcriptional activator in the vanSR regulator within the vanl glycopeptide resistance gene cluster. $\mathrm{H}$ : resistant strains selected at high copper ion exposure concentrations that gradually increased up to $1 / 10$ MIC level; L: resistant strains selected at low mental ion exposure concentrations about $1 / 100$ MIC

\section{The hereditary stability of antibiotic resistance in evolved E. coli strains}

Hereditary antibiotic resistance in the evolved strains exposure to sublethal concentration of metal and antibiotic co-contaminations is of great significance for human health and environmental safety (Lv et al. 2014; Zhang et al. 2018b). Figure 5 showed ciprofloxacin-, tetracycline-, and chloramphenicol- resistant MICs after 5 culture cycles in LB broth over 5 days. Results indicate that the resistance levels to tetracycline and chloramphenicol were significantly decreased by a relatively low sublethal concentration of copper ion or the relatively high sublethal concentration of the co-contaminations (Fig. 5b, c). Ciprofloxacin's resistance levels increased significantly with copper ions and tetracycline treatment, except for the relatively low sublethal concentration of copper ion (Fig. 5a).

\section{Discussions}

The present data analysis showed that sublethal concentration of copper ions and tetracycline co-contamination could enrich de novo resistant mutants to increase the corresponding antibiotics' mutation frequencies. A previous study indicated that environmental pollutions such as metals, biocides, and organometallics could promote the transmission of mobile genetic elements (MGE) through a co-selection mechanism (Kohanski et al. 2010), thereby contributing to antibiotic resistance, our research results are consistent with previous reports. Deficient concentrations of antibiotics (Kohanski et al. 2010; Gullberg et al. 2011), heavy metals (Li et al. 2019), disinfectants (Kohanski et al. 2010), and disinfection by-products (DBPs) (Lv et al. 2014; Li et al. 2016) could promote bacteria evolving antibiotic resistance were also reported. Therefore, together with previous reports, we hypothesized that the presence of ARB in varied sublethal concentrations of copper ions and tetracycline environments are partially understood through the selective effects of co-contaminated environmental chemicals, and the coselection of co-contaminations driven $E$. coli evolved antibiotic resistance via co-resistance or cross-resistance.

Additionally, many studies proposed that, environmental pollutions, such as metal, can accelerate ARG dissemination (Kohanski et al. 2010; Zhang et al. 2018a). This study suggests that heavy metals and residual antibiotics could result in long-term stress on antibiotic resistance populations.

The MIC determination was performed to investigate phenotypic evidence of resistance to antibiotics. The MICs were analyzed to evaluate the bacterial resistance. E. coli displayed the highest resistance to sublethal concentrations of copper ions and tetracycline co-contaminated treatments. The relatively high sublethal concentration of co-contamination was the most toxic compared with the relatively low sublethal or copper ions or tetracycline alone (Fig. 3). It is clear that the environment in which organisms exist affects their

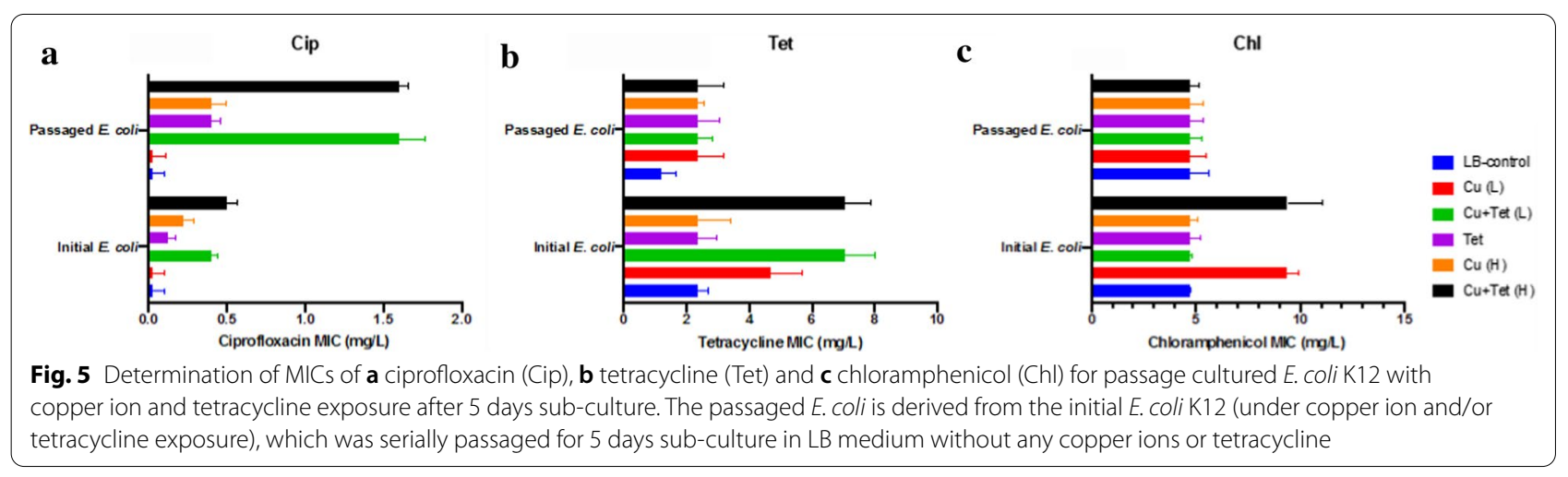


susceptibility to antibiotics, confirmed by the selection and enrichment of antibiotic-resistant organisms without antibiotic exposure (Kohanski et al. 2010). Two different changes were respectively identified on $m d t F$ and vanRI genes in the LB-control strain, could involve in the sub-culture environment. Common sources of environmental stress include metal ions, especially copper and zinc, which at low concentrations are essential for the function of normal bacterial cells and are necessary for some metalloproteins; however, they are toxic at high levels (Lemire et al. 2013). These metals provide selective stress for metal resistance at more elevated levels, which in turn is driven by genetic and physiological links (Fig. 4). Therefore, sublethal concentrations of copper ions and tetracycline co-contamination resulted in copper dose-dependent alteration in resistance mutation rates for polymyxin B (Fig. 2a) and chloramphenicol (Fig. 2b); the higher the level of copper concentration in the compounds, the higher chance of polymyxin B or chloramphenicol resistance; copper ion treatment alone was similar can be explained. In environments contaminated with copper and tetracycline, the enrichment of antibiotic-resistant organisms may be the selection of organisms on chromosomes or plasmids that carry resistance genes for both drugs (Table 2 and Fig. 4).

Previous reports suggested that chromosomal mutation is the primary way and mechanism for bacteria to acquire ARGs, such as base substitution or frameshift in specific genes (Kohanski et al. 2010). Several research reports suggest that sublethal concentrations (far below the MIC) of antibiotics can also induce mutation rate increases for bacteria, for example, in our study, sublethal concentrations of copper ions and tetracycline co-contaminated treatments critically enhance the resistant mutation rates of polymyxin $\mathrm{B}$ and chloramphenicol $(\mathrm{P}<0.05)$ (Fig. $2 \mathrm{a}$, b). Furthermore, these mutations appear at a high frequency due to the relatively low fitness-cost (Gullberg et al. 2011). This phenomenon indicated that the sublethal concentration of antibiotics is adequate at acquiring ARGs. Hence, this may explain that with a sublethal concentration of copper ions and tetracycline exposure, selected strains demonstrated clinically relevant resistance to ciprofloxacin, erythromycin, polymyxin B, tetracycline, and chloramphenicol with a significant increase in MICs.

The $m d t F$ is a multi-antibiotic inner membrane transporter for the mdtEF-TolC efflux complex. It is a resistance nodulation division (RND) type efflux pump in $E$. coli, with vital homology to $a c r B$, but is generally expressed at a low level in clinical isolates. A previous study indicated that overexpressed pumps could induce susceptibility to erythromycin (Bohnert et al. 2007). All Gram-negative strains have genes for efflux pumps that are the part of the RND family. These pumps effectively develop resistance to multiform complex mixtures, for example, antibiotics, dyes, and other xenobiotics, even multidrug resistance (MDR) (Jellen-Ritter and Kern 2001; Webber et al. 2005). The various MICs of most of the antibiotics were involved in detecting a single point mutation; this single-point mutation increases the resistance to exposed antibiotics but reduces the resistance to another antibiotic (Bohnert et al. 2007). These facts allow us to explain why the phenotype and genotype are inconsistent in this study.

VanRI gene is a regulatory activator of transcription in vanSR regulators within the vanI glycopeptide resistance gene cluster (ARO:3003728), a glycopeptide resistance gene conferring antibiotic resistance via molecular bypass. The $m d t F$ and vanRI mutations might contribute to developing ciprofloxacin, chloramphenicol, and polymyxin B resistance in E. coli (Figs. 2 and 3 ). The above findings indicate that at the relatively high copper ions sublethal contamination (below $1 / 10$ of the MIC) with tetracycline in polluted environments could increase mutation frequency. In this study, the number and uniqueness of genetic alterations were different from mutants caused by varied sublethal of copper ions and tetracycline co-contaminated environments; phenotypes and genotypes showed significant inconsistency.

The evolution and dissemination of antibiotic resistance are accelerated through the acquiring of ARGs via de novo mutation. Genetic mutations are regarded as significant pathways that contribute to the evolution of antibiotic resistance (Nolivos et al. 2019). The attenuation, persistence, and enrichment of ARGs or ARB in the environment are ecological evolution processes stimulated by diverse circumstances (Andersson and Hughes 2011).

The results of the hereditary stability of antibiotic resistance in evolved E. coli strains may be explained by the contribution of genotypic-phenotypic discrepancies (Beaber et al. 2004; Corona and Martinez 2013). Strains subjected to antibiotics, whether by increased or decreased resistance level, could relate to phenotypic changes (not-inheritable resistance) and genetic changes (inheritable resistance). Evolved strains considerably raised ciprofloxacin resistance and matched the initial E. coli $\mathrm{K} 12$ and the control strains; this may have been obtained via genetic changes, based on the wholegenome sequencing analysis. In terms of the adaptive evolution of resistant strains, the stable hereditary features of co-contaminations-induced ciprofloxacin resistance could pose a potential public health threat. In this study, ciprofloxacin resistance can be stably passed on to offspring by evolved strains; this hereditary stability could cause continued antibiotic resistance development. 
This study demonstrates a synergistic effect between sublethal doses of antibiotic and ionic copper, both individually and in combination, on stable mutations that lead to evolution of antibiotic resistant bacteria. Evidence was provided that sublethal concentrations of copper ions and tetracycline co-contaminated treatment could lead to significant increase in the evolution of polymyxin B and chloramphenicol resistant mutations. With sublethal concentration of copper ions and tetracycline exposure, the MICs of selected strains resistance to ciprofloxacin, erythromycin, polymyxin $\mathrm{B}$, tetracycline and chloramphenicol increase from 1 to 32 folds. In addition, two genetic changes involved in the antibiotic resistance gene were determined, that is $m d t F$ and vanRI. Hereditary antibiotic resistance of the evolved strains showed that the evolved resistance can be lost over subsequent generations, though not always. These results might contribute to understand the emergence and dissemination of antibiotic resistance in our environment.

\section{Supplementary Information}

The online version contains supplementary material available at https://doi. org/10.1186/s13568-020-01173-6.

Additional file 1: Text S1. Minimum inhibitory concentrations (MICs) determination. Fig S1. The MIC of Copper ions to wild-type E. coli K12.

\section{Acknowledgments}

This work was supported by the National Key Research and Development Program of China (Grant Number: 2016YFC0503601) from Ministry of Science and Technology of the People's Republic of China.

\section{Authors' contributions}

$J L$ and $Z Y$ conceived and designed research. $J L$ conducted experiments. IP and GZ contributed new reagents or analytical tools. JL, ZY, IP analyzed data. $J L$ wrote the manuscript. All authors read and approved the manuscript.

\section{Funding}

This study is financial supported by National Key R\&D Program of China (2016YFC0503601).

\section{Ethics approval and consent to participate}

All applicable international, national, and/or institutional guidelines for the care and use of animal were followed.

\section{Competing interests}

The authors declare that the research was conducted in the absence of any commercial or financial relationships that could be construed as a potential competing interest.

\footnotetext{
Author details

${ }^{1}$ College of Resources and Environment, University of Chinese Academy of Sciences, 19 A Yuquan Road, Shijingshan District, Beijing 100049, China. ${ }^{2}$ Ministry of Agriculture and Rural Affairs, Agro-Environmental Protection Institute, Tianjing 300191, China.
}

Received: 4 December 2020 Accepted: 24 December 2020 Published online: 07 January 2021

\section{References}

Alonso A, Sanchez P, Martinez JL (2001) Environmental selection of antibiotic resistance genes. Environ Microbiol 3:1-9

Andersson DI, Hughes D (2011) Persistence of antibiotic resistance in bacterial populations. FEMS Microbiol Rev 35:901-911

Baker-Austin C, Wright MS, Stepanauskas R, Mcarthur JV (2006) Co-selection of. antibiotic and metal resistance. Trends Microbiol 14:176-182

Beaber JW, Hochhut B, Waldor MK (2004) SOS response promotes horizontal dissemination of antibiotic resistance genes. Nature 427:72-74

Berg J, Tom-Petersen A, Nybroe O (2005) Copper amendment of agricultural soil selects for bacterial antibiotic resistance in the field. Lett Appl Microbiol 40:146-151

Bohnert JA, Schuster S, Fahnrich E, Trittler R, Kern WV (2007) Altered spectrum of. multidrug resistance associated with a single point mutation in the Escherichia coli RND-type MDR efflux pump YhiV (MdtF). J Antimicrob Chemother 59:1216-1222

Brockhurst MA, Colegrave N, Rozen DE (2011) Next-generation sequencing as a tool to study microbial evolution. Mol Ecol 20:972-980

Chen S, Li X, Sun G, Zhang Y, Su J, Ye J (2015) Heavy metal induced antibiotic resistance in bacterium LSJC7. Int J Mol Sci 16:23390-23404

Corona F, Martinez JL (2013) Phenotypic resistance to antibiotics. Antibiotics (Basel) 2:237-255

Gullberg E, Cao S, Berg OG, Ilbäck C, Sandegren L, Hughes D, Andersson DI (2011) Selection of resistant bacteria at very low antibiotic concentrations. PLoS Pathogens 7(7):e1002158

Gullberg E, Albrecht LM, Karlsson C, Sandegren L, Andersson DI (2014) Selection of a multidrug resistance plasmid by sublethal levels of antibiotics and heavy metals. MBio 5:e01918-e11914

Hau SJ, Frana T, Sun J, Davies PR, Nicholson TL (2017) Zinc Resistance within swine-associated methicillin-resistant staphylococcus aureus isolates in the United States is associated with multilocus sequence type lineage. Appl Environ Microbiol. https://doi.org/10.1128/AEM.00756-17

Henriques I, Tacao M, Leite L, Fidalgo C, Araujo S, Oliveira C, Alves A (2016) Co-selection of antibiotic and metal(loid) resistance in gram-negative epiphytic bacteria from contaminated salt marshes. Mar Pollut Bull 109:427-434

Huang Changgan ZL, Liping YU, Jinzhu C, Xiaoyue L (2004) A study on pollution of environment by copper and its phytoremediation in dexing copper mine. Acta Agriculturae Universitatis Jiangxiensis 26(4):902-904

Imran M, Das KR, Naik MM (2019) Co-selection of multi-antibiotic resistance in bacterial pathogens in metal and microplastic contaminated environments: An emerging health threat. Chemosphere 215:846-857

Jellen-Ritter AS, Kern WV (2001) Enhanced expression of the multidrug efflux pumps AcrAB and AcrEF associated with insertion element transposition in Escherichia coli mutants selected with a fluoroquinolone. Antimicrob Agents Chemother 45:1467-1472

Khan S, Beattie TK, Knapp CW (2017) The use of minimum selectable concentrations (MSCs) for determining the selection of antimicrobial resistant bacteria. Ecotoxicology 26:283-292

Kohanski MA, Depristo MA, Collins JJ (2010) Sublethal antibiotic treatment leads to multidrug resistance via radical-induced mutagenesis. Mol Cell 37:311-320

Lemire JA, Harrison JJ, Turner RJ (2013) Antimicrobial activity of metals: mechanisms, molecular targets and applications. Nat Rev Microbiol 11:371-384

Li D, Zeng S, He M, Gu AZ (2016) Water disinfection byproducts induce antibiotic. resistance-role of environmental pollutants in resistance phenomena. Environ Sci Technol 50:3193-3201

Li X, Gu AZ, Zhang Y, Xie B, Li D, Chen J (2019) Sub-lethal concentrations of heavy. metals induce antibiotic resistance via mutagenesis. J Hazard Mater 369:9-16

Lu X, Gao Y, Luo J, Yan S, Rengel Z, Zhang Z (2014) Interaction of veterinary antibiotic tetracyclines and copper on their fates in water and water hyacinth (Eichhornia crassipes). J Hazard Mater 280:389-398

Lv L, Jiang T, Zhang S, Yu X (2014) Exposure to mutagenic disinfection byproducts leads to increase of antibiotic resistance in Pseudomonas aeruginosa. Environ Sci Technol 48:8188-8195

Martinez JL (2008) Antibiotics and antibiotic resistance genes in natural environments. Science 321:365-367

Nolivos S, Cayron J, Dedieu A, Page A, Delolme F, Lesterlin C (2019) Role of AcrAB-TolC multidrug efflux pump in drug-resistance acquisition by plasmid transfer. Science 364:778-782 
Pan X, Qiang Z, Ben W, Chen M (2011) Residual veterinary antibiotics in swine manure from concentrated animal feeding operations in Shandong Province, China. Chemosphere 84:695-700

Peltier E, Vincent J, Finn C, Graham DW (2010) Zinc-induced antibiotic resistance in activated sludge bioreactors. Water Res 44:3829-3836

Poole K (2017) At the nexus of antibiotics and metals: the impact of Cu and Zn on antibiotic activity and resistance. Trends Microbiol 25:820-832

Povolo VR, Ackermann M (2019) Disseminating antibiotic resistance during treatment. Science 364:737-738

Pruden A, Pei R, Storteboom H, Carlson KH (2006) Antibiotic resistance genes as. emerging contaminants: studies in northern Colorado. Environ Sci Technol 40:7445-7450

Seiler C, Berendonk TU (2012) Heavy metal driven co-selection of antibiotic resistance in soil and water bodies impacted by agriculture and aquaculture. Front Microbiol 3:399

Stepanauskas R, Glenn TC, Jagoe CH, Tuckfield RC, Lindell AH, Mcarthur JV (2005) Elevated microbial tolerance to metals and antibiotics in metalcontaminated industrial environments. Environ Sci Technol 39:3671-3678

Vikesland PJ, Pruden A, Alvarez PJJ, Aga D, Burgmann H, Li XD, Manaia CM, Nambi I, Wigginton K, Zhang T, Zhu YG (2017) Toward a comprehensive strategy to mitigate dissemination of environmental sources of antibiotic resistance. Environ Sci Technol 51:13061-13069
Wang FH, Qiao M, Su JQ, Chen Z, Zhou X, Zhu YG (2014) High throughput profiling of antibiotic resistance genes in urban park soils with reclaimed water irrigation. Environ Sci Technol 48:9079-9085

Webber MA, Talukder A, Piddock $\sqcup$ (2005) Contribution of mutation at amino acid. 45 of AcrR to acrB expression and ciprofloxacin resistance in clinical and veterinary Escherichia coli isolates. Antimicrob Agents Chemother 49:4390-4392

Zhang Y, Gu AZ, Cen T, Li X, He M, Li D, Chen J (2018a) Sub-inhibitory concentrations of heavy metals facilitate the horizontal transfer of plasmidmediated antibiotic resistance genes in water environment. Environ Pollut 237:74-82

Zhang Y, Gu AZ, Xie S, Li X, Cen T, Li D, Chen J (2018b) Nano-metal oxides induce antimicrobial resistance via radical-mediated mutagenesis. Environ Int 121:1162-1171

Zhu YG, Johnson TA, Su JQ, Qiao M, Guo GX, Stedtfeld RD, Hashsham SA, Tiedje JM (2013) Diverse and abundant antibiotic resistance genes in Chinese swine farms. Proc Natl Acad Sci U S A 110:3435-3440

\section{Publisher's Note}

Springer Nature remains neutral with regard to jurisdictional claims in published maps and institutional affiliations.

\section{Submit your manuscript to a SpringerOpen ${ }^{\odot}$ journal and benefit from:}

- Convenient online submission

- Rigorous peer review

- Open access: articles freely available online

- High visibility within the field

- Retaining the copyright to your article

Submit your next manuscript at $\boldsymbol{\nabla}$ springeropen.com 